$\begin{array}{cl}\text { PRAMANA } & \text { (C) Indian Academy of Sciences } \\ \begin{array}{c}\text { journal of } \\ \text { physics }\end{array} & \begin{array}{l}\text { Vol. } 71, \text { No. } 2 \\ \text { August } 2008 \\ \text { pp. } 403-412\end{array}\end{array}$

\title{
Quenching dynamics of a quantum $X Y$ spin-1/2 chain in the presence of transverse field by the application of a generalized Landau-Zener formula
}

\author{
VICTOR MUKHERJEE ${ }^{1, *}$, UMA DIVAKARAN ${ }^{1}$, AMIT DUTTA $^{1}$ \\ and DIPTIMAN SEN ${ }^{2}$ \\ ${ }^{1}$ Department of Physics, Indian Institute of Technology, Kanpur 208 016, India \\ ${ }^{2}$ Centre for High Energy Physics, Indian Institute of Science, Bangalore 560 012, India \\ ${ }^{*}$ Corresponding author. E-mail: victor@iitk.ac.in
}

\begin{abstract}
In this paper we review the quenching dynamics of a quantum $X Y$ spin-1/2 chain in the presence of a transverse field, when the transverse field or the anisotropic interaction is quenched at a slow but uniform rate. We also extend the results to the cases in which the system starts with any arbitrary initial condition as opposed to the initial fully magnetically aligned state which has been extensively studied earlier. The evolution is non-adiabatic in the time interval when the parameters are close to their critical values, and is adiabatic otherwise. The density of defects produced due to nonadiabatic transitions is calculated by mapping the many-particle system to an equivalent Landau-Zener problem. We show that in one dimension the density of defects in the final state scales as $1 / \sqrt{\tau}$ irrespective of the initial condition, where $\tau$ is the quenching time-scale. However, the magnitude of density of defects is found to depend on the initial condition.
\end{abstract}

Keywords. Transverse $X Y$ model; quantum phase transition; Landau-Zener.

\section{PACS Nos 73.43.Nq; 05.70.Jk; 75.10.Jm}

Wide applicability of quantum dynamics in different branches of science has led to a recent upsurge in the study of dynamical behaviour of different quantum systems. The possibility of experimental realizations of non-equilibrium strongly correlated quantum systems [1] has paved the way for rigorous theoretical investigations [2-4] of the dynamics of various model Hamiltonians when swept across the quantum critical points. One of the possible scenarios is Kibble-Zurek mechanism [5] which predicts the functional dependence of the density of defects on the quenching rate in a system passing through a quantum critical point. When a system crosses a quantum critical point, relaxation time $\left(\xi_{T}\right)$ diverges, thus forcing the system to evolve non-adiabatically, thereby producing kinks or oppositely oriented spins. If the energy gap $(\Delta)$ varies as $t / \tau$, correlation length $(\xi)$ grows as $\xi \sim(t / \tau)^{-\nu}$ and $\xi_{T} \sim(t / \tau)^{-\nu z}$, where $\nu, z$ are quantum critical exponents. According to KibbleZurek mechanism the time $\hat{t}$ at which non-adiabatic effects set in is given by the 
condition $(\hat{t} / \tau)^{-\nu z}=\Delta / \frac{\mathrm{d}}{\mathrm{d} t}(\Delta)$ yielding $\hat{t}=\tau^{-\nu z /(\nu z+1)}$. Therefore, the length scale $(\hat{\xi})$ varies as $\hat{\xi} \sim(\hat{t})^{1 / z} \sim(\tau)^{-\nu /(\nu z+1)}$. The defect density of the final state is therefore given by $n \sim 1 / \hat{\xi}^{d} \sim 1 / \tau^{\nu d /(\nu z+1)}$, where $d$ is the spatial dimensionality. For a transverse Ising model in $d=1$ we get $\nu=z=1$ which gives $n \sim 1 / \sqrt{\tau}$. Recently, rigorous calculations using LZ formula [6] has shown that $1 / \sqrt{\tau}$ scaling behaviour of the kink density is valid in the case of quenching dynamics of transverse Ising and $X Y$ spin chain [5,7-11]. We should also mention that some other studies on gapless systems and two-dimensional Kitaev model show violation of KibbleZurek prediction [12].

It is well-known that the transverse Ising and transverse $X Y$ chain Hamiltonians can be reduced to two-level problems in Fourier space following fermionization via Jordan-Wigner transformation [13]. The LZ formula turns out to be very helpful in calculating the transition probability to the excited state of a two-level system when a parameter in the Hamiltonian is changed at a slow but uniform rate. In this work we review the results of quenching dynamics of a transverse $X Y$ spin chain where the initial state is fully magnetically aligned $[7,8]$. We also find out the defect density in the final state when the initial state is partially polarized using a generalized version of the LZ formula. We show that the kink density still scales as $1 / \sqrt{\tau}$ for large $\tau$ in $d=1$ though the magnitude of the density of kinks depends on the initial ground state.

Our system of interest is an anisotropic spin- $1 / 2 X Y$ model with transverse field given by

$$
H=-\frac{1}{2} \sum_{n}\left(J_{x} \sigma_{n}^{x} \sigma_{n+1}^{x}+J_{y} \sigma_{n}^{y} \sigma_{n+1}^{y}+h \sigma_{n}^{z}\right),
$$

where the $\sigma$ 's are Pauli spin matrices satisfying the usual commutation relations. $h$ denotes the transverse magnetic field, whereas $J_{x}$ and $J_{y}$ are strengths of ferromagnetic couplings along $x$ - and $y$-directions respectively. In this work, $J_{x}, J_{y}$ and $h$ are chosen to be non-random. In order to explore the phase diagram of the Hamiltonian, we initially choose time-independent values of the parameters. In the limit $J_{y}=0$, Hamiltonian in eq. (1) reduces to the transverse Ising model [14], while for $J_{x}=J_{y}$ it describes an isotropic $X Y$ model in a transverse field [13]. The Hamiltonian in eq. (1) can be exactly diagonalized using the Jordan-Wigner transformation which maps a system of spin- $1 / 2$ 's to a system of spinless fermions $[13,15,16]$. The Jordan-Wigner transformation of spins to fermions is given by

$$
\begin{aligned}
& c_{n}=\left(\prod_{j=-\infty}^{n-1} \sigma_{j}^{z}(-1)^{n} \sigma_{n}^{-},\right. \\
& c_{n}^{\dagger}=\left(\prod_{j=-\infty}^{n-1} \sigma_{j}^{z}\right)(-1)^{n} \sigma_{n}^{+},
\end{aligned}
$$

where $\sigma_{n}^{ \pm}=\left(\sigma_{n}^{x} \pm i \sigma_{n}^{y}\right) / 2$ are the spin raising (lowering) operators. The relation between $\sigma_{n}^{z}$ and fermion operators is given as $\sigma_{n}^{z}=2 c_{n}^{\dagger} c_{n}-1$; thus the presence (absence) of a fermion at site $n$ implies a spin-up (down) state. In terms of fermion 
creation and annihilation operators, the above Hamiltonian can be rewritten in Fourier space with a periodic boundary condition as

$$
\begin{aligned}
H= & -\sum_{k>0}\left\{\left[\left(J_{x}+J_{y}\right) \cos k+h\right]\left(c_{k}^{\dagger} c_{k}+c_{-k}^{\dagger} c_{-k}\right)\right. \\
& \left.+i\left(J_{x}-J_{y}\right) \sin k\left(c_{k}^{\dagger} c_{-k}^{\dagger}-c_{-k} c_{k}\right)\right\} .
\end{aligned}
$$

This Hamiltonian being quadratic in the fermion operators can be diagonalized using the standard Bogoliubov transformation. The gap in the excitation spectrum is given by $[13,15]$

$$
\epsilon_{k}=\left[h^{2}+J_{x}^{2}+J_{y}^{2}+2 h\left(J_{x}+J_{y}\right) \cos k+2 J_{x} J_{y} \cos 2 k\right]^{1 / 2} .
$$

We probe the regions at which the gap given in eq. (4) vanishes. The vanishing of the gap at $h=\mp\left(J_{x}+J_{y}\right)$ at wave vectors $k=0$ and $\pi$ signals a quantum phase transition from a quantum paramagnetic phase to a ferromagnetically ordered phase. This transition belongs to the universality class of the transverse Ising model $[14,15]$ and is therefore referred to as the 'Ising' transition. The gapless line $J_{x}=J_{y}$ $\left(|h|<\left|J_{x}+J_{y}\right|\right)$ separates two phases ferromagnetically ordered along $x$ - and $y$ directions, denoted by $\mathrm{FM}_{x}$ and $\mathrm{FM}_{y}$ respectively, and the corresponding phase transition is called the 'anisotropic transition'. When $J_{x}>J_{y}$ the ferromagnetic ordering is in the $x$-direction, and hence it is in $\mathrm{FM}_{x}$ phase while it is the other way round in the $\mathrm{FM}_{y}$ phase. Each ferromagnetically ordered phase is further divided into a commensurate and an incommensurate region with the incommensurate wave vector $k_{0}$ given by

$$
\cos k_{0}=-\frac{h\left(J_{x}+J_{y}\right)}{4 J_{x} J_{y}} .
$$

On the anisotropic phase boundary $J_{x}=J_{y}$, the incommensurate wave vector therefore picks up a value $k_{0}=\cos ^{-1}\left(-h / 2 J_{x}\right)$. The boundary between these two regions inside a ferromagnetic phase is given by the relation $h /\left(J_{x}+J_{y}\right)= \pm\left(1-\gamma^{2}\right)$, with $\gamma \equiv\left(J_{x}-J_{y}\right) /\left(J_{x}+J_{y}\right)$, as shown by the thick dashed lines in figure 1 . The points $J_{x}=J_{y}$ and $h= \pm 2 J_{y}$ are multicritical points. It will be discussed later that the quenching dynamics show a different behaviour when the system passes through those points.

To study the quenching dynamics of the above model two quenching schemes have been proposed and studied in recent years. In the 'transverse quenching' $[7,9]$ scheme the transverse field $(h)$ is quenched as $h=t / \tau$. On the other hand, in the 'anisotropic quenching' [8] case, the ferromagnetic coupling $\left(J_{x}\right)$ along the $x$ direction is quenched as $J_{x}=t / \tau$. In either cases $t$ varies from $-\infty$ to $\infty$. Clearly in both the cases the initial state is fully magnetically aligned.

By Jordan-Wigner transformation followed by Fourier transformation as explained above we project the Hamiltonian $H_{k}(t)$ to the two-dimensional subspace spanned by $|0\rangle$ and $|k,-k\rangle$ for studying the dynamics of transverse quenching. The Hamiltonian takes the form

$$
\begin{aligned}
H_{k}(t)= & -\left[h+\left(J_{x}+J_{y}\right) \cos k\right] I_{2} \\
& +\left[\begin{array}{cc}
h+\left(J_{x}+J_{y}\right) \cos k & i\left(J_{x}-J_{y}\right) \sin k \\
-i\left(J_{x}-J_{y}\right) \sin k & -h-\left(J_{x}+J_{y}\right) \cos k
\end{array}\right],
\end{aligned}
$$

Pramana - J. Phys., Vol. 71, No. 2, August 2008 
Victor Mukherjee et al

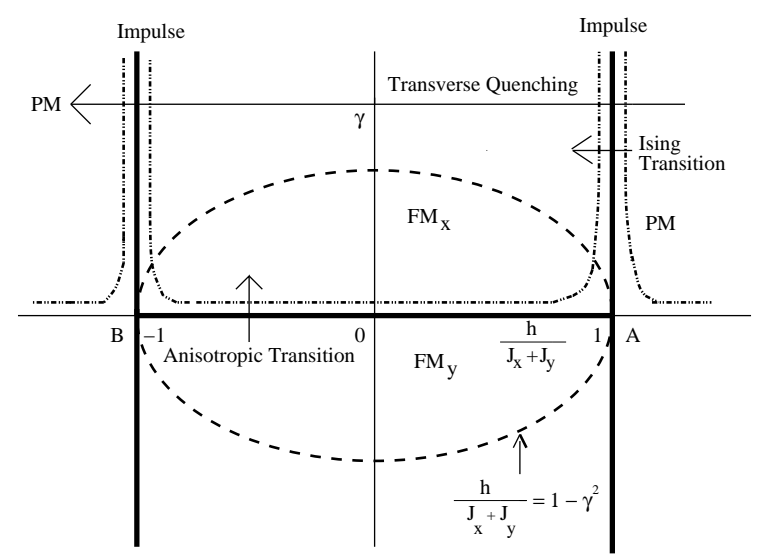

Figure 1. The phase diagram of the anisotropic $X Y$ model in a transverse field in the $h /\left(J_{x}+J_{y}\right)-\gamma$ plane. Ising transitions occur along the vertical bold lines given by $h /\left(J_{x}+J_{y}\right)= \pm 1$. The system is also gapless on the horizontal bold line $J_{x}=J_{y}$ for $|h|<\left|J_{x}+J_{y}\right| . \mathrm{FM}_{x}\left(\mathrm{FM}_{y}\right)$ is a long-range ordered phase with ferromagnetic ordering in the $x(y)$ direction. The thick dashed line marks the boundary separating the commensurate and incommensurate ferromagnetic phases. System evolves non-adiabatically in the impulse regions bounded by the thin dotted lines when the field $h$ is quenched from $-\infty$ to $\infty$. Multicritical points are denoted by the points A and B.

where $I_{2}$ denotes the $2 \times 2$ identity matrix. A state in this subspace can be represented as $\psi_{k}(t)=u_{k}(t)|0\rangle+v_{k}(t)|k,-k\rangle$, where the probability amplitudes $u_{k}(t)$ and $v_{k}(t)$ are time-dependent. The initial condition is given by $u_{k}(-\infty)=1$ and $v_{k}(-\infty)=0$. The time evolution of a generic state is governed by the Schrödinger equation

$$
i \partial_{t} \psi_{k}(t)=H_{k}(t) \psi_{k}(t)
$$

The off-diagonal term of the projected Hamiltonian, $\Delta=\left(J_{x}-J_{y}\right) \sin k$, represents the interaction term between the two time-dependent levels. In the case of transverse quenching the time-dependent energy levels are given by $E_{1,2}=$ $\pm\left[h(t)+\left(J_{x}+J_{y}\right) \cos k\right]$. The Schrödinger equation given above is identical to the Landau-Zener problem of a two-level system. Using the Landau-Zener transition formula, the transition probability of excitations at the final time is given by $[6]$

$$
p_{k}=\mathrm{e}^{-2 \pi \tilde{\gamma}}
$$

where $\tilde{\gamma}=\Delta^{2} /\left|\frac{\mathrm{d}}{\mathrm{d} t}\left(E_{1}-E_{2}\right)\right|$. Equivalently, $p_{k}$ determines the probability that the system remains in the initial state $|0\rangle$ at the final time. For the transverse quenching case, one can use the relation in eq. (7) to obtain $p_{k}$ as a function of the anisotropy term $J_{x}-J_{y}$ and $\tau$ as [7]

$$
p_{k}=\mathrm{e}^{-\pi \tau\left(J_{x}-J_{y}\right)^{2} \sin ^{2} k} .
$$


Quenching dynamics of a quantum XY spin-1/2 chain

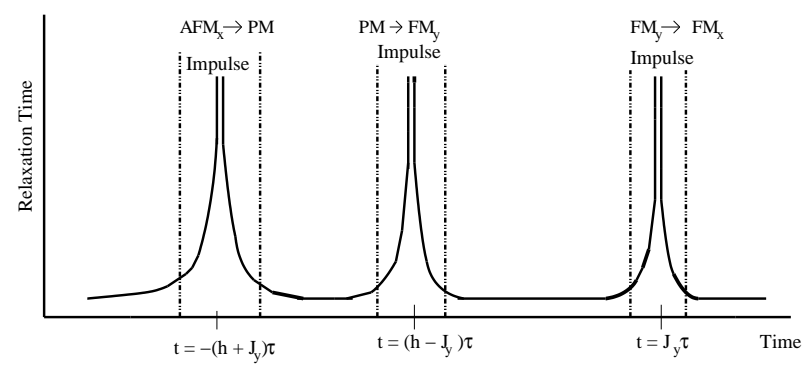

Figure 2. Schematic diagram showing the divergence of the relaxation time of the quantum Hamiltonian at the quantum critical points. As discussed in the text, the dotted vertical lines denote the regions ('impulse regions') where the system is forced to evolve non-adiabatically.

This leads to an expression for the density of kinks $n$ generated due to non-adiabatic transitions,

$$
n=\int_{0}^{\pi} \frac{\mathrm{d} k}{\pi} p_{k} \simeq \frac{1}{\pi \sqrt{\tau}\left|J_{x}-J_{y}\right|} .
$$

Equation (9) shows that the kink density decreases as $1 / \sqrt{\tau}$ for large $\tau$ as predicted by the Kibble-Zurek theory and proved by Cherng and Levitov in the case of transverse quenching [7].

We shall now focus on the anisotropic quenching scheme where the interaction term $J_{x}(t)=t / \tau$ is changed from $-\infty$ to $\infty$, with $J_{y}$ and $h$ held fixed at some positive values. Initial state of the system is fully antiferromagnetically polarized in the $x$-direction. As $J_{x}$ increases from $-\infty$, the system goes through Ising and anisotropic critical lines at different points of time [8] as depicted in the static phase diagram of the model shown in figure 1 . The adiabatic and non-adiabatic regions of time evolution are shown schematically in figure 2. In this scheme, Hamiltonian $H_{k}(t)$ has time-dependent off-diagonal terms, which renders LZ mechanism apparently inapplicable here. However, by an appropriate unitary transformation [8] to a basis in which $\sigma_{x}$ is diagonal, a LZ type of Hamiltonian with time-independent off-diagonal terms can be constructed in the case of anisotropic quenching also. The new Hamiltonian is given by

$$
\begin{aligned}
H_{k}^{\prime}(t)= & -\left[h+\left(J_{x}+J_{y}\right) \cos k\right] I_{2} \\
& +\left[\begin{array}{cc}
J_{x}(t)+J_{y} \cos 2 k+h \cos k & J_{y} \sin 2 k+h \sin k \\
J_{y} \sin 2 k+h \sin k & -J_{x}(t)-J_{y} \cos 2 k-h \cos k
\end{array}\right] .
\end{aligned}
$$

Therefore, the anisotropic quenching is now placed on the same footing as the transverse quenching case. Naturally, a general state vector can be expressed as a linear combination of $|e\rangle_{1 k}$ and $|e\rangle_{2 k}$,

$$
\left|\psi_{k}(t)\right\rangle=C_{1 k}(t)\left|e_{1 k}\right\rangle+C_{2 k}(t)\left|e_{2 k}\right\rangle,
$$

where $|e\rangle_{1 k}$ and $|e\rangle_{2 k}$ are the eigenvectors of the Hamiltonian $H_{k}^{\prime}(t)$. The initial condition in the anisotropic quenching case happens to be $C_{1 k}(-\infty)=1$ and 
Victor Mukherjee et al

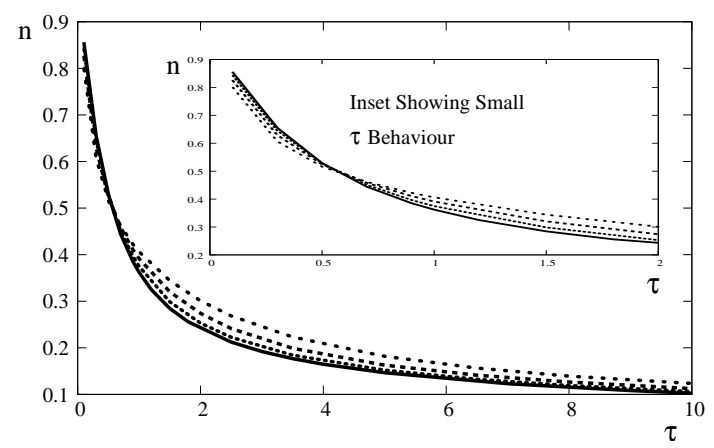

Figure 3. Variation of kink density $n$ with $\tau$ as obtained analytically for $h=0.2,0.4,0.6,0.8$ (from bottom to top), with $J_{y}=1$. For large $\tau, n$ increases with increasing $h$, whereas for small $\tau$ it decreases for increasing $h$.

$C_{2 k}(-\infty)=0$. So the anisotropic non-adiabatic transition probability now turns out to be a function of $J_{y}$ and $h$, and is given by

$$
p_{k}=\left|C_{1 k}(\infty)\right|^{2}=\mathrm{e}^{-\pi \tau\left(J_{y} \sin 2 k+h \sin k\right)^{2}} .
$$

As in the transverse quenching case, we now measure the density of kinks given by

$$
n=\int_{0}^{\pi} \frac{\mathrm{d} k}{\pi} p_{k}=\int_{0}^{\pi} \frac{\mathrm{d} k}{\pi} \mathrm{e}^{-\pi \tau\left(J_{y} \sin 2 k+h \sin k\right)^{2}} .
$$

In figure 3, the plot of the kink density as a function of the quenching time $\tau$ is shown, which clearly shows that $n \propto 1 / \sqrt{\tau}$ for large values of $\tau$. This finding supports the prediction of the Kibble-Zurek mechanism even in the anisotropic quenching case. The approximate analytical form of $n$ for large $\tau$ is given by [8]

$$
n \simeq \frac{4 J_{y}}{\pi \sqrt{\tau}\left(4 J_{y}^{2}-h^{2}\right)} .
$$

Figure 3 shows that this approximate form matches the exact result perfectly in the limit of large $\tau$. The kink density $n$ increases with increasing field $h$ as shown in figure 3. On the other hand, for small $\tau$, the density $n$ is found to decrease with increasing $h$. The cross-over from the small $\tau$ to the large $\tau$ behaviour occurs around a typical quenching time $\tau_{0}=\ln 2 /\left[\pi\left(J_{y} \sin 2 k+h \sin k\right)^{2}\right]$. Equation (13) shows a diverging kink density for $2 J_{y}=h$. We have already mentioned that the point $J_{x}=J_{y}=|h| / 2$ is a multicritical point. Interestingly, when the system crosses the multicritical point, the kink density shows a much slower fall given as $1 / \tau^{1 / 6}$

The LZ formula used here is applicable only when the initial condition is such that at $t \rightarrow-\infty$ the system is in fully magnetically aligned state, i.e. $C_{1 k}(-\infty)=1 \forall k$. However, in order to solve the dynamics when the system does not start from a fully aligned state we need to extend the LZ formula to the case of arbitrary initial conditions. Let us consider two energy levels $\epsilon_{1}(t)$ and $\epsilon_{2}(t)$ which are moving with time as shown in figure 4 , with a time-independent coupling between them. The Hamiltonian is given by 
Quenching dynamics of a quantum $X Y$ spin-1/2 chain

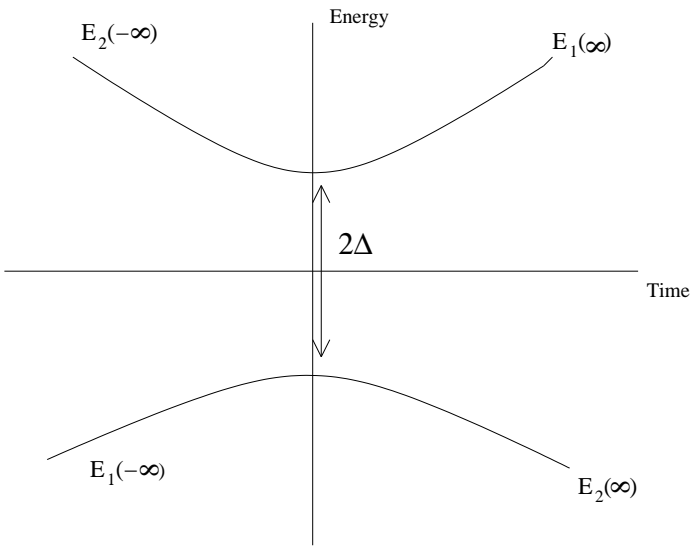

Figure 4. Landau-Zener energy levels. $E_{1}(t), E_{2}(t)$ are the eigenvalues of $H$.

$$
\begin{aligned}
H= & H_{0}+H_{\text {int }}=\left(\epsilon_{1}(t)|1(t)\rangle\left\langle 1(t)\left|+\epsilon_{2}(t)\right| 2(t)\right\rangle\langle 2(t)|\right) \\
& +\Delta(|1(t)\rangle\langle 2(t)|+| 2(t)\rangle\langle 1(t)|),
\end{aligned}
$$

where

$$
H_{0}=\epsilon_{1}(t)|1(t)\rangle\left\langle 1(t)\left|+\epsilon_{2}(t)\right| 2(t)\right\rangle\langle 2(t)|
$$

and

$$
H_{\text {int }}=\Delta(|1(t)\rangle\langle 2(t)|+| 2(t)\rangle\langle 1(t)|) .
$$

$\epsilon_{j}(t)$ and $|j(t)\rangle(j=1,2)$ denote the instantaneous eigenenergy and eigenstate of $H_{0}(t)$ respectively. We assume a linear variation of $\epsilon_{1}(t)-\epsilon_{2}(t)$ given by $\epsilon_{1}(t)-$ $\epsilon_{2}(t)=\alpha(t)$, where $\alpha$ is a positive constant. Coupling between the two states is given by $H_{\text {int }}$ in which $\Delta$ is time-independent. The level crossing takes place at $t=0$ where the energy gap is also minimum, given by $2 \Delta$.

Defining a general state vector as $|\Psi(t)\rangle=\bar{C}_{1}(t)|1(t)\rangle+\bar{C}_{2}(t)|2(t)\rangle$ one can immediately write down the evolution equation for the probability amplitudes $\bar{C}_{1}(t)$ and $\bar{C}_{2}(t)$ as

$$
\begin{aligned}
i \frac{\mathrm{d}}{\mathrm{d} t} \bar{C}_{1}(t) & =\epsilon_{1}(t) \bar{C}_{1}(t)+\Delta \bar{C}_{2}(t), \\
i \frac{\mathrm{d}}{\mathrm{d} t} \bar{C}_{2}(t) & =\epsilon_{2}(t) \bar{C}_{2}(t)+\Delta \bar{C}_{1}(t) .
\end{aligned}
$$

We make a convenient choice of the variables as

$$
\begin{aligned}
& \bar{C}_{1}(t)=C_{1}(t) \mathrm{e}^{-i \int_{-\infty}^{t} \mathrm{~d} t^{\prime} \epsilon_{1}\left(t^{\prime}\right)} \\
& \bar{C}_{2}(t)=C_{2}(t) \mathrm{e}^{-i \int_{-\infty}^{t} \mathrm{~d} t^{\prime} \epsilon_{2}\left(t^{\prime}\right)}
\end{aligned}
$$


and finally introduce a variable

$$
C_{2}(t)=\mathrm{e}^{\left[-\frac{i}{2} \int_{\infty}^{t} \mathrm{~d} t^{\prime}\left(\epsilon_{1}\left(t^{\prime}\right)-\epsilon_{2}\left(t^{\prime}\right)\right)\right]} U(t)
$$

to arrive at an equation for the evolution operator $U$ given as

$$
\frac{\mathrm{d}^{2}}{\mathrm{~d} z^{2}} \bar{U}(z)+\left(n+\frac{1}{2}-\frac{1}{4} z^{2}\right) \bar{U}(z)=0
$$

where $z=\mathrm{e}^{-\pi / 4 i} \alpha^{1 / 2} t$ and $n=i \Delta^{2} / \alpha$. Two independent solutions of eq. (21), known as Weber's differential equation, are given by $D_{n}(z)$ and $D_{-n-1}(i z)$ where

$$
D_{n}(z)=2^{\frac{1}{2} n+\frac{1}{4}} z^{-\frac{1}{2}} W_{\frac{1}{2} n+\frac{1}{4},-\frac{1}{4}}\left(\frac{1}{2} z^{2}\right)
$$

and

$$
\begin{aligned}
& W_{k, m}(z) \\
& \sim \mathrm{e}^{-\frac{1}{2} z} z^{k}\left[1+\sum_{n=1}^{\infty} \frac{\left(m^{2}-\left(k-\frac{1}{2}\right)^{2}\right)\left(m^{2}-\left(k-\frac{3}{2}\right)^{2}\right) \cdots\left(m^{2}-\left(k-n+\frac{1}{2}\right)^{2}\right)}{n ! z^{n}}\right]
\end{aligned}
$$

for large values of $z[28]$. Putting these values in $D_{n}(z)$ and $D_{-n-1}(i z)$ we find that, in the limit $R \rightarrow \infty$,

$$
D_{n}\left(R \mathrm{e}^{-i \pi / 4}\right) \simeq \mathrm{e}^{\frac{\pi \Delta^{2}}{4 \alpha}} \mathrm{e}^{i\left(\frac{R^{2}}{4}+\frac{\Delta^{2}}{\alpha} \ln R\right)}
$$

and in the same limit $D_{-n-1}\left(i R \mathrm{e}^{-\frac{\pi}{4} i}\right)=0$, where $R=\alpha^{1 / 2} t$. Therefore, in the limit $R \rightarrow \infty$ this gives $\left|D_{n}\left(R \mathrm{e}^{-i \pi / 4}\right)\right|^{2}=\mathrm{e}^{\pi \Delta^{2} / 2 \alpha}$. For convenience, let us take some arbitrary value of $C_{2}(\infty)$ as the initial condition. The limiting value of eq. (22) gives

$$
\left|C_{2}(\infty)\right|^{2}=|B|^{2} \mathrm{e}^{\pi \Delta^{2} / 2 \alpha},
$$

where $|B|^{2}$ is a constant to be determined. Equation (23) yields

$$
|B|^{2}=\left|C_{2}(\infty)\right|^{2} \mathrm{e}^{-\pi \Delta^{2} / 2 \alpha} .
$$

On the other hand, in the limit $R \rightarrow-\infty$, it can be shown that $D_{n}\left(R \mathrm{e}^{-i \pi / 4}\right)=$ $\mathrm{e}^{\frac{3 \pi \Delta^{2}}{4 \alpha}} \mathrm{e}^{i\left(\frac{R^{2}}{4}+\frac{\Delta^{2}}{\alpha} \ln |R|\right)}$ and $D_{-n-1}\left(i R \mathrm{e}^{-i \pi / 4}\right)=0$. Using this result along with eq. (24), it can be shown that

$$
\left|C_{2}(-\infty)\right|^{2}=\left|C_{2}(\infty)\right|^{2} \mathrm{e}^{-2 \pi \gamma},
$$

where $\gamma=\frac{\Delta^{2}}{\frac{\mathrm{d}}{\mathrm{d} t}\left|\epsilon_{1}(t)-\epsilon_{2}(t)\right|}$. In addition to $\left|C_{2}(\infty)\right|^{2}$, if we consider the contribution coming from the spins that were already in the excited state at $t \rightarrow+\infty$, i.e. $\left|C_{1}(\infty)\right|^{2}$, we get

$$
\left|C_{2}(-\infty)\right|^{2}=\left|C_{2}(\infty)\right|^{2} \mathrm{e}^{-2 \pi \gamma}+\left|C_{1}(\infty)\right|^{2}\left(1-\mathrm{e}^{-2 \pi \gamma}\right)
$$


In the same spirit, one can arrive at the relation

$$
\left|C_{1}(\infty)\right|^{2}=\left|C_{1}(-\infty)\right|^{2} \mathrm{e}^{-2 \pi \gamma}+\left|C_{2}(-\infty)\right|^{2}\left(1-\mathrm{e}^{-2 \pi \gamma}\right)
$$

Hence for the special case which is used in previous calculation with $\left|C_{1}(-\infty)\right|^{2}=1$ and $\left|C_{2}(-\infty)\right|^{2}=0$ we get $\left|C_{1}(\infty)\right|^{2}=\mathrm{e}^{-2 \pi \gamma}$.

Now we shall apply this generalized Landau-Zener formula for the quenching dynamics of the above spin chain when quenched from $t \rightarrow-\infty$ to $t \rightarrow \infty$ for any arbitrary initial condition, i.e., $\left|C_{1 k}(-\infty)\right|^{2}$ is not necessarily equal to 1 for all $k$, implying that the initial state is not perfectly magnetically ordered. This means that in the case of transverse quenching some spins are aligned opposite to the direction of magnetic field at $t \rightarrow-\infty$, and in the case of anisotropic quenching, perfect antiferromagnetic alignment is not present in the system even when $J_{x} \rightarrow$ $-\infty$. Equation (27) shows that the probability of non-adiabatic transition is given by $p_{k}=\left|C_{1 k}(-\infty)\right|^{2} \mathrm{e}^{-2 \pi \gamma}+\left|C_{2 k}(-\infty)\right|^{2}\left(1-\mathrm{e}^{-2 \pi \gamma}\right)$ and therefore the kink density $n=\frac{1}{2 \pi} \int_{-\pi}^{\pi}\left[\left|C_{1 k}(-\infty)\right|^{2} \mathrm{e}^{-2 \pi \gamma}+\left|C_{2 k}(-\infty)\right|^{2}\left(1-\mathrm{e}^{-2 \pi \gamma}\right)\right] \mathrm{d} k$. As shown previously, by an appropriate unitary transformation the anisotropic quenching can be brought to the same footing as the transverse quenching. So the same conclusion holds good for both types of quenching, even at the multicritical point.

\section{Conclusions}

We have studied the adiabatic quantum dynamics of an anisotropic $X Y$ spin-1/2 chain in a transverse field when the parameters of the Hamiltonian are quenched at a steady and finite rate. We have looked into the cases in which the system does not necessarily start from its initial ground state. We have also calculated the kink density, and found it to satisfy Kibble-Zurek mechanism irrespective of the initial state.

\section{References}

[1] M Greiner, O Mandel, T Esslinger, T W Hänsch and I Bloch, Nature (London) 415, $39(2002)$

T Stöferle, H Moritz, C Schori, M Köhl and T Esslinger, Phys. Rev. Lett. 92, 130403 (2004)

V M H Ruutu, V B Eltsov, A J Gill, T W B Kibble, M Krusius, Yu G Makhlin, B Placais, G E Volovik and Wen Xu, Nature (London) 382, 334 (1996)

C Bäuerle, Yu M Bunkov, S N Fisher, H Godfrin and G R Pickett, Nature (London) 382, 332 (1996)

R Carmi, E Polturak and G Koren, Phys. Rev. Lett. 84, 4966 (2000).

[2] K Sengupta, S Powell and S Sachdev, Phys. Rev. A69, 053616 (2004)

[3] P Calabrese and J Cardy, J. Stat. Mech: Theory Expt. P04010 (2005)

[4] A Das, K Sengupta, D Sen and B K Chakrabarti, Phys. Rev. B74, 144423 (2006)

[5] W H Zurek, U Dorner and P Zoller, Phys. Rev. Lett. 95, 105701 (2005)

[6] S Suzuki and M Okada, in Quantum annealing and related optimization methods edited by A Das and B K Chakrabarti (Springer-Verlag, Berlin, 2005) p. 185

[7] R W Cherng and L S Levitov, Phys. Rev. A73, 043614 (2006) 
[8] V Mukherjee, U Divakaran, A Dutta and D Sen, Phys. Rev. B76, 174303 (2007)

[9] J Dziarmaga, Phys. Rev. Lett. 95, 245701 (2005)

[10] A Polkovnikov, Phys. Rev. B72, 161201(R) (2005)

[11] U Divakaran and A Dutta, J. Stat. Mech. P11001 (2007)

[12] A Polkovnikov and V Gritsev, arXiv:0706.0212

[13] E Lieb, T Schultz and D Mattis, Ann. Phys. (NY) 16, 407 (1961)

[14] P Pfeuty, Ann. Phys. (NY) 57, 79 (1970)

[15] J E Bunder and R H McKenzie, Phys. Rev. B60, 344 (1999)

[16] J B Kogut, Rev. Mod. Phys. 51, 659 (1979)

[17] T W B Kibble, J. Phys. A9, 1387 (1976)

[18] W H Zurek, Nature (London) 317, 505 (1985); Phys. Rep. 276, 177 (1996)

[19] B Damski and W H Zurek, Phys. Rev. A73, 063405 (2006)

[20] J Dziarmaga, Phys. Rev. B74, 064416 (2006)

[21] T Caneva, R Fazio and G E Santoro, Phys. Rev. B76, 144427 (2007)

[22] F M Cucchietti, B Damski, J Dziarmaga and W H Zurek, Phys. Rev. A75, 023603 (2007)

[23] R Schützhold, M Uhlmann, Y Xu and U R Fischer, Phys. Rev. Lett. 97, 200601 (2006)

[24] T Kadowaki and H Nishimori, Phys. Rev. E58, 5355 (1998)

[25] Quantum annealing and related optimization methods edited by A Das and B K Chakrabarti (Springer-Verlag, Berlin, 2005)

[26] E Barouch and B M McCoy, Phys. Rev. A3, 786 (1971)

[27] C Zener, Proc. R. Soc. London Ser. A137, 696 (1932)

L D Landau and E M Lifshitz, Quantum mechanics: Non-relativistic rheory, 2nd ed. (Pergamon Press, Oxford, 1965)

[28] E T Whittaker and G N Watson, A course of modern analysis, 4th ed. (Cambridge At The University Press, 1958) p. 343 